\title{
Accurate Prediction of Kinematic Viscosity of Biodiesels and their Blends with Diesel Fuels
}

Masoud Mehrizadeh Hadi Nikbin-Fashkacheh Nima Zand $\underline{\text { Adel Najafi-Marghmaleki }}$

First published: 13 September 2020

https://doi.org/10.1002/aocs.12421

\begin{abstract}
Viscosity of mixtures of biodiesels (admixtures) and mixtures of biodiesel/diesel (blends) is a important parameter for determining their combustion behavior. There is no universal and general model for prediction of viscosity of these systems at different conditions. Hence, developing simple, accurate, and general models for prediction of viscosity of these systems is of great importance. In this work, three computer-based models named multilayer perceptron neural network (MLP-NN), radial basis function optimized by particle swarm optimization (PSO-RBF), and adaptive neuro fuzzy inference system optimized by hybrid approach (Hybrid-ANFIS) were developed for prediction of viscosity of blends and admixtures. A number of 966 experimental data covering wide ranges of influencing parameters were utilized to develop the models. The accuracy of predictions of the developed models was examined by using different statistical quality measure approaches as well as comparing their results with the predictions of literature models. Results showed that the developed models present accurate predictions and are superior to the literature models. The predictions of PSO-RBF model were also better than HybridANFIS and MLP-NN models.
\end{abstract}

